resse et Docteur Daniele Marceau et le procureur général de Québec, mis en cause.

2 Came B. The Last Goodbye. Nancy B. exercises her right to die. MacLean's February 24, 1992. p 50.

3 Benrubi GI. Euthanasia - The need for procedural safeguards. N Engl J Med 1992; 326: 197-9.

\section{Four-centre study of anaesthetic outcomes}

\section{To the Editor:}

We would like to comment on the very interesting and topical series of papers written by $\mathrm{M}$. Cohen et al. ${ }^{1-3}$ They are to be congratulated for the incredible amount of time and effort which they have expended in collecting and analyzing data on 37,665 anaesthetics. However, we do have some concerns regarding certain aspects of the data collection and interpretation.

Data were gathered using a combination of chart review and patient interview. The authors carefully note at least 15 factors which may effect the validity of the data collected by these techniques. Of importance, many of these elements differed among the various institutions studied. Other potential problems with the data collection involve the process of performing patient interviews. As all practising clinicians are aware, patients are often unreliable historians, especially for comfort-related problems. Indeed, the actual act of asking whether a patient has a specific symptom may itself increase the incidence of findings, due to auto-suggestion. Patients may also be influenced by external factors such as suggestions by surgical or nursing staff (which may be institutionspecific) and preconceived notions of "usual anaesthetic complications" (particularly sore throat and nausea/vomiting). Furthermore, some patients will clearly have preexisting dementia or psychological unreliability and, as such, must be excluded from this type of data collection. Since the patient interviews were an integral part of the process assessing the frequency of postoperative complications, the "disturbing" incidence of sore throat, nausea, headache and backache in outpatients alluded to in the third study ${ }^{3}$ may be at least partly attributed to the above factors. It would be interesting to know the frequency of these complications which were graded as severe, since considering just the severe symptoms may diminish the effect of some of the preceding potential sources of bias.

Although it is difficult to know the exact impact of the plethora of factors which may bias the data collected in these studies, it is very likely that, at least in combination, they could effect the reliability and validity of that data. Since the conclusions of any study are limited by the quality of the initial data, it is probably inappropriate to derive anything more than very preliminary conclusions from the data of the current studies.

To help assess the validity of the methods used in these studies, the investigators quantified the reliability of two specific data collection techniques: the postoperative patient interview questionnaire, and the panel assessment of causation of ten hypothetical cases. Although the former method appeared to be reliable, with interrater agreement of $>95 \%$, the latter technique exhibited poor interrater agreement, with a kappa value of only 0.38 . Despite this latter observation, the authors conclude that "the ratings of actual cases could be reliably carried out by the audit committees at each hospital." This conclusion, is not justified since the investigators did not quantitate the reliability of the causation assessment for real cases and there is no reason to anticipate that the assessment of real cases would be more reliable than that of the hypothetical cases. To justify the above conclusion, the authors refer to the higher degrees of reliability obtained using similar types of assessments reported in previous studies. We fail to understand the validity of this line of reasoning. Indeed, it would be interesting to discover why the interrater agreement of the current study differed from that previously reported.

The conclusions of any study ultimately depend upon the statistical validity. As the number of comparisons performed on the same body of data increases, the likelihood of any comparison becoming significant due to chance alone also increases. Accordingly, as more and more subsets of data are analyzed the acceptable $P$ value for a 1:20 chance of alpha error decreases, in a manner analagous to the Bonferroni correction for multiple $t$ tests. ${ }^{4}$ In the second and third papers, several large tables are shown representing comparisons of many different variables. Statistical significance was set at $P \leq 0.05$ in the second study and the "more conservative value of $P \leq$ $0.01^{\text {" }}$ in the third. Probably even lower $P$ values of $<0.001$ or less would have been more appropriate for most of these tables, based on the Bonferroni correction of $P=0.05 / n$ ( $n=$ number of comparisons). The investigators' choice of $P$ value may have contributed to the occurrence of some unexpected correlations, such as the association between preoperative hypertension and difficult intubation. Such a relationship has no apparent biological plausibility and, as such, may represent an artifact of the statistical analysis.

Finally, occurrence screening studies of this sort are generally not considered an end in themselves but are useful in planning future prospective, randomized clinical 
trials. These studies must be performed carefully and analyzed conservatively if one is to avoid making premature, incorrect conclusions. In particular, it will be important to resist the temptation to use this type of analysis for comparing the "quality" of anaesthesia among different hospials, unless one can control for all the institutionspecific factors which may influence patient outcome.

\author{
Peter H. Norman \\ M.D. Daley \\ Department of Anaesthesia \\ Toronto General Hospital \\ University of Toronto
}

\section{REFERENCES}

1 Cohen MM, Duncan PG, Tweed WA, et al. The Canadian four-centre study of anaesthetic outcomes: I. Description of methods and populations. Can J Anaesth 1992; 39: 420-9.

2 Cohen MM, Duncan PG, Pope WDB, et al. The Canadian four-centre study of anaesthetic outcomes: II. Can outcomes be used to assess the quality of anaesthesia care? Can J Anaesth 1992; 39: 430-9.

3 Duncan PG, Cohen MM, Tweed WA, Biehl D, Pope $W D B$, Merchant $R N$. The Canadian four-centre study of anaesthetic outcomes: III. Are anaesthetic complications predictable in day surgical practice? Can J Anaesth 1992; 39: 440-8.

4 Glantz SM. Primer of Biostatistics. 2nd ed. New York: McGraw-Hill, 1987.

\section{REPLY}

We thank Drs. Norman and Daley for taking the time to write about our study. There are several points to which we wish to respond.

The first issue raised relates to patient interviews. We disagree that "patients are ofien unreliable historians." It is more likely that health professionals do not ask questions in a manner which will solicit the desired information. Questions tend not to be asked in a systematic fashion during day-to-day clinical practice and comments such as "is everything alright" are very vague. It appears to be true that prompting the patient elicits higher reporting of rates of discomfort symptoms. There are several reasons for this. First patients do not want to "bother" busy doctors and nurses with complaints, even sometimes if they are troublesome and, as the authors point out, patients often expect to undergo some umpleasantness when having an operation. If asked "do you (or did you) have any problems with your anaesthetic," patients may only remember the most severe or unexpected symptoms or may be too inhibited to mention problems. Alternatively they may be so pleased to have "made it through" that lesser complaints are ignored. General questions concerning comfort and well-being lack the sensitivity to detect morbidity and negative answers cannot ensure that a given outcome was absent. In order to determine the actual rate of discomfort symptoms (whether severe or not), asking the patient is the best (and often the only) method. In improving the quality of anaesthesia care, a mild degree of discomfort should not be the goal - elimination of symptoms altogether should be the ultimate aim.

In our study we used a systematic approach to patient interviews. Every patient was asked questions in a very similar manner and patients who could not understand the questions or who were too ill were excluded from the analysis. Thus we do not think that the results we achieved in the third paper were due to factors alluded to by Drs. Norman and Daley. However, we do note in the paper that the response rate to the interviews was very low and non-representativeness of the patients may have given rise to certain results. For example it may be that only the very sick patients were at home to receive phone calls the day after surgery thus explaining the high rate of symptoms in this population.

Drs. Norman and Daley also allude to the difficulty in performing studies of this type (specifically relating to reliability and validity) and point out that only "very preliminary conclusions" should be derived. We could not agree more. Although not necessary, we elected for various reasons to publish a separate paper on the methodological issues arising from such a study. First, we wanted to identify for anaesthetists how to perform this type of study, since "outcome" studies are rare in the field. Second, the conclusions of similar studies done in virtually every department (under the rubric of "audit" or " $Q A$ ") are often accepted without challenge, but lack validity for the reasons we discuss. Finally, by describing our methods in some detail, we challenge other researchers to design studies which overcome some of these difficulties. Just as anaesthetists are not using the same drugs and protocols as they did even five years ago, clinical epidemiologists continuously update their study methods. In many large, multisite studies, the researchers have not described their protocols in nearly the detail which we did. This does mean that they did not confront similar issues but rather only that they went unrecognized.

With respect to the low kappa value across physician case reviewers, we agree that the value was less than desirable. We did not evaluate the reliability of the "causation assessment for real cases" because we used a consensus process. This was for pragmatic reasons: it was virtually impossible to get 12 busy clinician volunteers to review all cases, and we did not have adequate funds for training sessions for the reviewers. Future studies will have to pay case reviewers for their time and training. This was done in two studies quoted in the paper and would likely account for their high interrater reliability. How ever, despite the low kappa values we decided to use case reviewers to reflect actual clinical practice. Reviewers for morbidity/mortality rounds are rarely trained and usually only one person performs this function for each case. Yet in clinical practice, the "reliability" of this opinion is rarely questioned.

The next issue relates to the $P$ values used for setting statistical significance in this study. While we agree with the authors, we wish to point out that the $P$ values reported in the tables were those factors considered statistically significant at the $P$ less than or equal to 0.01 . The reader might infer that all the values reported were significant only at the 0.01 level, but that is the minimum which we accepted. Most of the values were much lower than this (see examples in the Table which correspond to Table VI in the third paper).' Not all statistical experts agree with the use of the Bonferroni correction for all situations. The correction tends to be too conservative if the number of comparisons is very high (i.e., no relationships 